\title{
A CLINICAL STUDY ON LINEAR DERMATOSES OCCURRING IN PAEDIATRIC AGE GROUP
}

Surya Prabha Paul Udaya Suriyan'1, Adikrishnan Swaminathan'2, Krishnakanth Muralidharan³, Murugan Sundaram4, Sudha Rangarajan ${ }^{5}$, Manu Vidhya Harikumar6, Mahalakshmi Veeraraghavan ${ }^{7}$

${ }_{1}$ Postgraduate Student, Department of Dermatology, Sri Ramachandra University, Chennai.

${ }^{2}$ Associate Professor, Department of Dermatology, Sri Ramachandra University, Chennai.

${ }^{3}$ Associate Professor, Department of Dermatology, Sri Ramachandra University, Chennai.

4 Professor, Department of Dermatology, Sri Ramachandra University, Chennai.

5 Professor, Department of Dermatology, Sri Ramachandra University, Chennai.

${ }^{6}$ Senior Resident, Department of Dermatology, Sri Ramachandra University, Chennai.

${ }^{7}$ HOD and Professor, Department of Dermatology, Sri Ramachandra University, Chennai.

ABSTRACT

\section{BACKGROUND}

Dermatoses affecting the skin exhibit various morphologies such as annular, arcuate, discoid, polycyclic, reticulate, target, confluent, guttate, stellate, digitate, linear, whorled, etc. The purpose of this study was to observe the prevalence, age and sex distribution, topography and the clinical morphological presentations of linear dermatoses occurring in paediatric age group.

\section{MATERIALS AND METHODS}

This is an observational study conducted at the dermatology outpatient department, Sri Ramachandra University, Chennai. They were clinically evaluated with detailed history including family history, history of presenting illness with complete physical examination and relevant investigations. Patients who fulfilled both the inclusion and exclusion criteria were included in this study after proper counselling and recording their assent and consents.

\section{RESULTS}

A total number of 30 patients showing evidence of linear dermatoses taken were included in this study. There was no sex predilection observed in this study, as it occurred equally in both sexes. Most commonly observed linear condition in this study was Lichen striatus. The other dermatoses observed in this study were linear lichen planus, inflammatory linear verrucous epidermal nevus, lichen nitidus, paederus dermatitis, hypomelanosis of Ito, linear and whorled nevoid hypermelanosis.

\section{CONCLUSION}

There has not been any similar studies on linear dermatoses as such. Its importance in dermatology cannot be overemphasised, but their unique morphology leads to early diagnosis which may help in prompt management of the condition.

\section{KEYWORDS}

Linear, Blaschko's Lines, Lichen Striatus, Paediatric.

HOW TO CITE THIS ARTICLE: Suriyan SPPU, Swaminathan A, Muralidharan K, et al. A clinical study on linear dermatoses occurring in paediatric age group. J. Evolution Med. Dent. Sci. 2017;6(89):6184-6188, DOI: 10.14260/jemds/2017/1344

\section{BACKGROUND \\ Objective}

Dermatoses affecting the skin exhibit various morphologies such as annular, arcuate, discoid, polycyclic, reticulate, target, confluent, guttate, stellate, digitate, linear, whorled, etc. Majority of the linear lesions follow the lines of Blaschko. The appearance of these conditions is helpful in clinching the diagnosis in various disorders. This study is an observational study based on dermatoses that occur in a linear pattern in paediatric age group as linearity allures the attention of the patients and their immediate family.

'Financial or Other Competing Interest': None.

Submission 28-08-2017, Peer Review 24-09-2017,

Acceptance 30-09-2017, Published 13-11-2017.

Corresponding Author:

Adikrishnan Swaminathan,

6th block, 3D Ceebros Park 2A,

Radhakrishnan Salai,

Valasaravakkam

Chennai-600087.

E-mail: adi_krish@yahoo.com

DOI: $10.14260 /$ jemds $/ 2017 / 1344$

The purpose of this study was to observe the prevalence, age and sex distribution, topography and the clinical morphological presentations of linear dermatoses occurring in paediatric age group.

\section{MATERIALS AND METHODS \\ Study Design}

The present study was an observational study.

\section{Study Setting}

Sri Ramachandra Medical College and Research Institute (Sri Ramachandra University), Porur, Chennai.

\section{Data Collection}

Inclusion criteria included patients in both sexes and those who are 17 and below 17 years of age. Exclusion criteria included Koebner's phenomenon and all patients above 17 years of age.[1] They were clinically evaluated with detailed history including family history, history of presenting illness with complete physical examination and relevant investigations. Patients who fulfilled both the inclusion and 
exclusion criteria were included in this study after proper counselling and recording the assent and consents.

\section{RESULTS}

A total number of 30 patients showing evidence of linear dermatoses were taken up in this study. There was no sex predilection observed in this study, as it occurred equally in both sexes [Figure 3]. The youngest age recorded was in a 10 month-old boy and the highest age was 17 years. Most commonly observed linear condition in this study was Lichen striatus [Figure 1]. Majority of patients showed unilateral distribution in a linear pattern, more often on the extremities, mainly the upper extremity [Figure 2]. These lesions were normally asymptomatic. There were no underlying defects or any other associated systemic abnormalities observed in this study. Other dermatoses observed in this study were Lichen striatus, Linear Lichen planus, inflammatory Linear verrucous epidermal nevus, Lichen nitidus, Paederus dermatitis, Hypomelanosis of Ito, Linear and Whorled nevoid hypermelanosis.
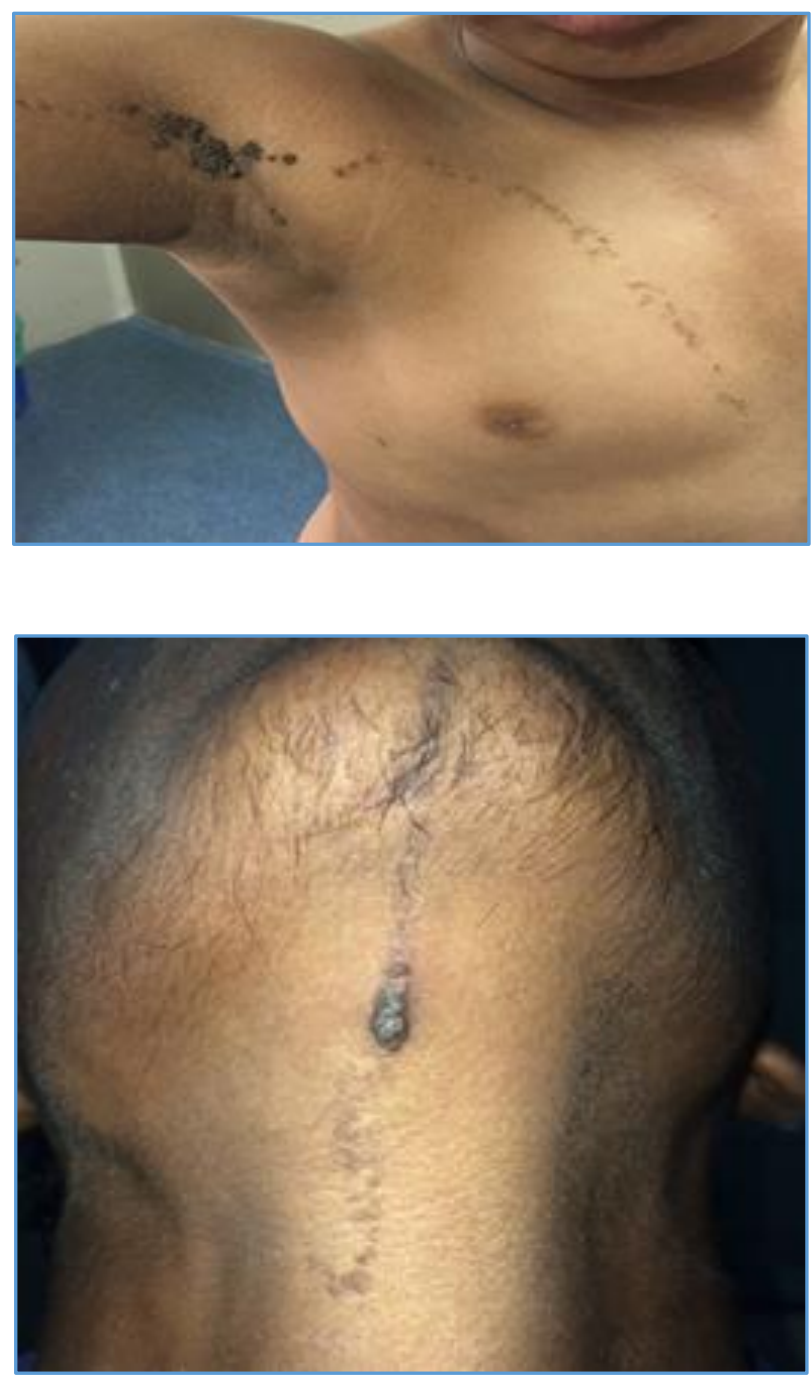

Picture 1. Inflammatory Linear Verrucous Epidermal Nevi

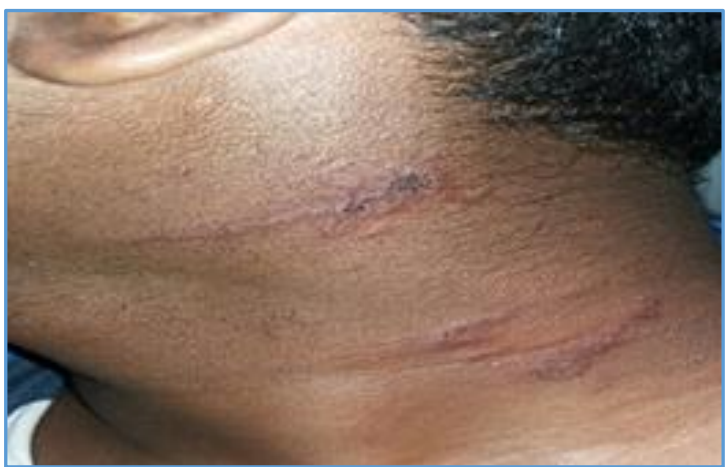

Picture 2. Paederus Dermatitis

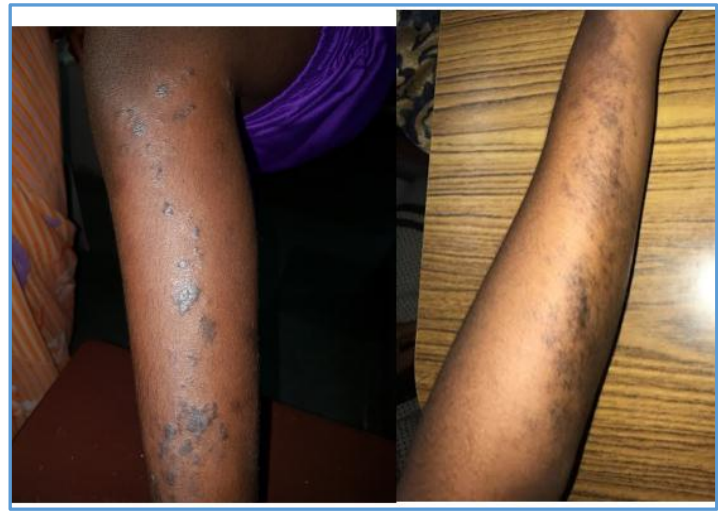

Picture 3. Linear Lichen Planus

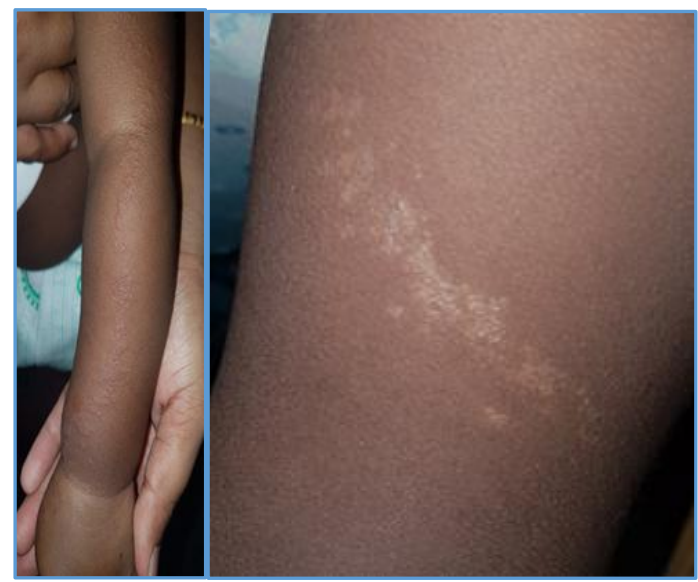

Picture 4. Lichen Striatus

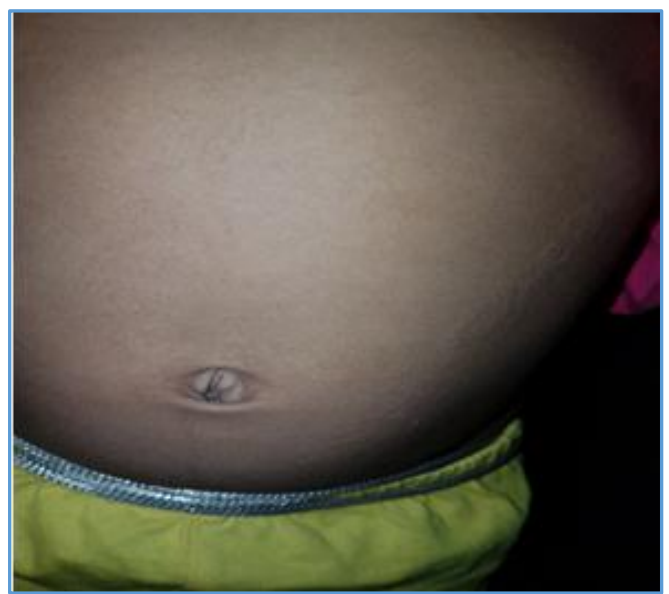

Picture 5. Lichen Nitidus 


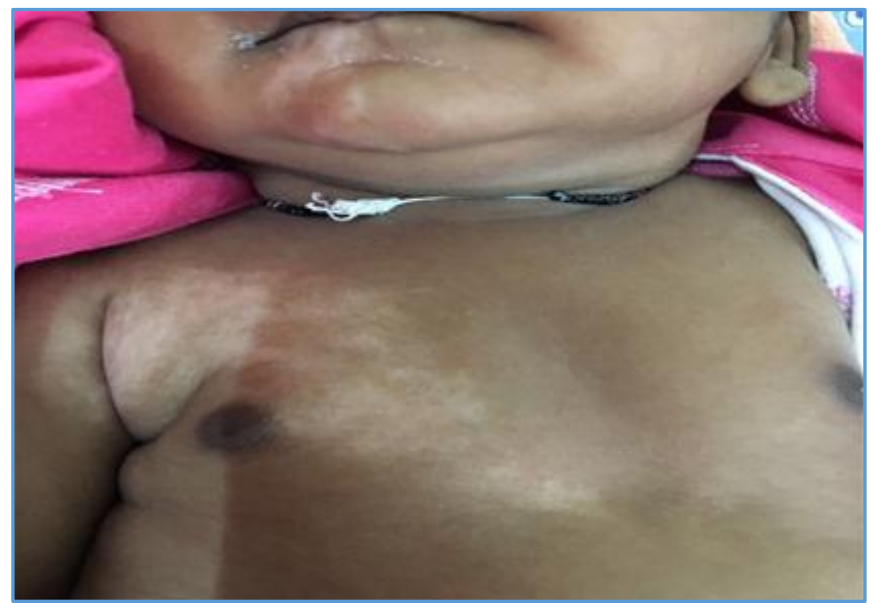

Picture 6. Hypomelanosis of Ito

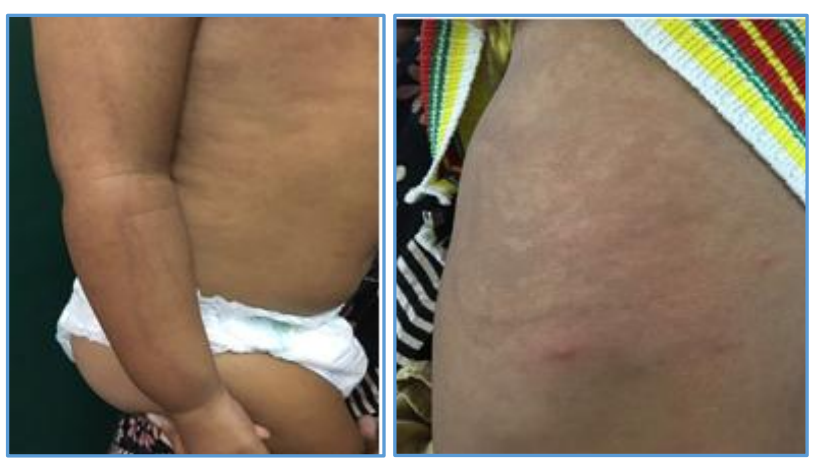

Picture 7. Linear and Whorled Nevoid Hypermelanosis

\section{DISCUSSION}

Linear dermatoses comprise either a single linear lesion or multiple lesions arranged in a linear pattern. Most of the lesions usually follow the Blaschko's lines. The lines of Blaschko represents the developmental growth pattern during epidermal cell migration. They are not in relation to any nervous, vascular or lymphatic structures. Typically, these lines are not visible; however, certain inherited and acquired diseases of the skin follow them. They follow various configurations such as V-shaped on the upper spine, S-shaped on the abdomen, inverted U-shaped on the breast area onto the upper arm, perpendicular lines in front and back of the lower extremities. They are less well-defined on the head and neck. Anatomical equivalent of Blaschko's lines has been described in teeth and eyes as well. They represent a form of human mosaicism. Mosaicism refers to two or more genetically distinct cell populations when present in an individual derived from a single zygote. Types of mosaicism are functional X-chromosome mosaicism, lethal autosomal mutations and non-lethal autosomal mutations. Causes are due to Lyonization, post-zygotic somatic mutations during early embryogenesis, gametic half-chromatid mutation occurring before fertilisation. These linear dermatoses following the lines of Blaschko are classified into genodermatoses like Incontinentia pigmenti, Focal dermal hypoplasia, X-linked hypohidrotic ectodermal dysplasia, Conradi-Hunermann syndrome, CHILD syndrome, Orofacial digital syndrome type 1, familial cutaneous amyloidosis Partington type, Segmental ash-leaf spot, Melanotic macules of McCune-Albright syndrome, Menkes syndrome; congenital or nevoid conditions such as Hypomelanosis of Ito, Nevus depigmentosus, Linear and whorled nevoid hypermelanosis, Inflammatory linear epidermal verrucous nevus, Linear porokeratosis of Mibelli, Nevus sebaceous of Jadassohn, Linear nevus comedonicus, Nevus corniculatus, Linear Darier's disease, Relapsing linear acantholytic dermatosis, Linear eccrine nevi, Syringocystadenoma papilliferum, Linear basal cell nevus, Unilateral nevoid basal cell carcinoma syndrome, Bart syndrome and acquired conditions such as Lichen striatus, Linear psoriasis, Linear lichen planus, Linear mucinoses and mycosis fungoides, Linear fixed drug eruption, Lupus erythematosus, Extragenital linear lichen sclerosus et atrophicus, Generalised lichenoid drug eruption, Linear atrophoderma of Moulin, Linear morphoea.[2] Other causes for occurrence in linear pattern includes configurations determined by course of blood vessels, lymphatics or nerve trunks, lesions following dermatomal pattern, lesions caused by external factors like plants, allergens, chemicals, lesions following infestations like cutaneous larva migrans, scabies, etc. [3]

In this study of 30 cases, most of the lesions were observed following the lines of Blaschko. It was observed equally in all divisions under paediatric age groups and it was observed more commonly in boys. $57 \%$ of patients were boys and the remaining $43 \%$ were girls. Most of the patients were asymptomatic with an exception being linear lichen planus patients who presented with complaints of itching. 17 cases of Lichen striatus, 5 cases of Linear Lichen planus, 2 cases of inflammatory linear verrucous epidermal nevus, Paederus dermatitis, Lichen nitidus and 1 case of Hypomelanosis of Ito and Linear and whorled nevoid hypermelanosis were encountered. Most common site of involvement was the upper limb (12 cases) followed by the trunk, lower limbs and face. The most common linear dermatological condition encountered was Lichen striatus. Lichen striatus is an uncommon self-limiting linear dermatosis with unknown aetiology and spontaneous regression. It primarily occurs in children from 5 - 15 years of age. The average age at diagnosis is 3 years.[4],[5] Females are affected more than males. Hauber et al reported a male predominance with maleto-female ratio of 3: 1.[6] They are usually asymptomatic with no predisposing factors. Though atopic diathesis is associated in $85 \%$ of these conditions, in this study none had any history or any family history of atopy. There were no associations with any other dermatoses. These lesions occur commonly on one arm or leg or on the neck, but may develop on the trunk, abdomen, buttocks or thighs.[4],[5] In this study, it was commonly seen on the upper limbs. Linear lichen planus was the second most common linear condition in this study with a total number of 5 cases. Lichen planus is a papulosquamous disease, in its classical presentation is characterised by pruritic violaceous papules, most commonly seen on the extremities.[7] In this study they were usually symptomatic, itching being the most common symptom. They initially developed as small, violaceous, hyperpigmented papules and plaques that progressed in a linear fashion following the Blaschko's lines. Most common site of distribution was the upper limb, similar to the other studies by Shankar Gouda Ireddy et al and Lakshmipriya Gurusamy et al.[8,9] Lichen planus is a commonly encountered dermatosis in clinical 
practice. Amongst the various morphological variants, linear lichen planus occurring along lines of Blaschko seems to be the uncommon mode of presentation. ${ }^{[10]}$ Inflammatory Linear Verrucous Epidermal Nevus was observed in 2 patients. They are a subset of epidermal nevi that were erythematous, inflamed and pruritic. These nevi is an unique variety of keratinocytic epidermal nevus, which exhibit both psoriasiform and inflammatory features. These lesions presented as hyperpigmented, verrucous papules and plaques distributed in a linear pattern. They were not present since birth, but became apparent later in life. Also, 2 cases of lichen nitidus were documented in this study. The lesions were asymptomatic, although one patient had pruritus. They developed as small pinpoint skin-coloured papules coalescing to form a plaque arranged in a linear distribution. Two cases of paederus dermatitis, a case of hypomelanosis of Ito and linear and whorled nevoid hypermelanosis were also documented. Hypomelanosis of Ito present as hypochromic unilateral or bilateral lesions in whorls, patches and streaks. $^{11}$ Neurological involvement is seen in $76 \%$ of patients, which present as seizures and mental retardation. Other associated features include ataxia, neuropathy, distal spinal muscular atrophy, torticollis, deafness, spina bifida occulta. In this study, there were no underlying defects or any other associations with hypomelanosis of Ito. Paederus dermatitis is a unique type of irritant contact dermatitis characterised by erythematous, vesicular or bullous lesions of sudden onset on exposed areas of the body. It is due to an insect belonging to the genus Paederus. This rove beetle neither bites or stings, but accidental brushing against or crushing the beetle on the skin on contact provokes the release of its fluid, which contains paederin, a potent vesicant. Diffuse erythematous and desquamative lesions, which predominantly occur on the upper body and face have been reported.[12] In this study it was seen on the face and the patient gave an acute history with complaints of burning sensation. Linear and whorled nevoid hypermelanosis is characterised by hyperpigmented macules along the Lines of Blaschko without preceding inflammation or atrophy. Lesions are distributed on the trunk and extremities sparing palms, soles and mucosae. The usual age of onset of hyperpigmentation occurs within the first few weeks of life, continues to progress for a year or two before stabilisation. There are very few case reports from India.[10] In this study, this condition was seen in a 2-year-old boy with the lesion occurring on the trunk.

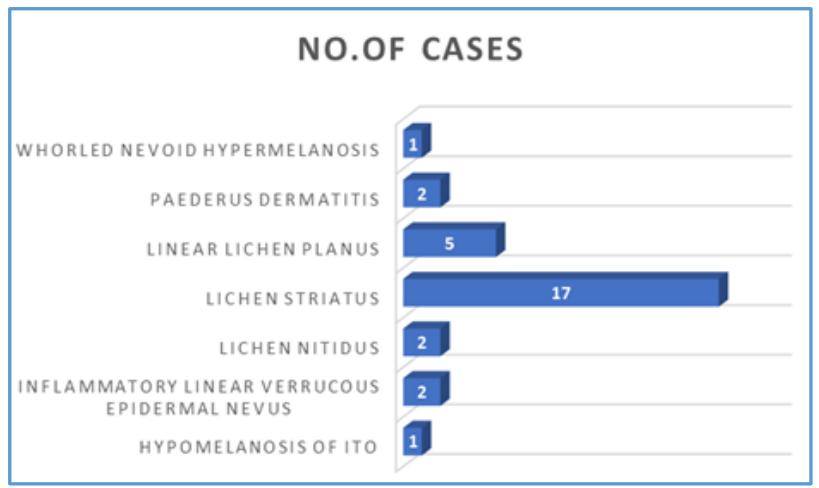

Figure 1. Prevalence Chart

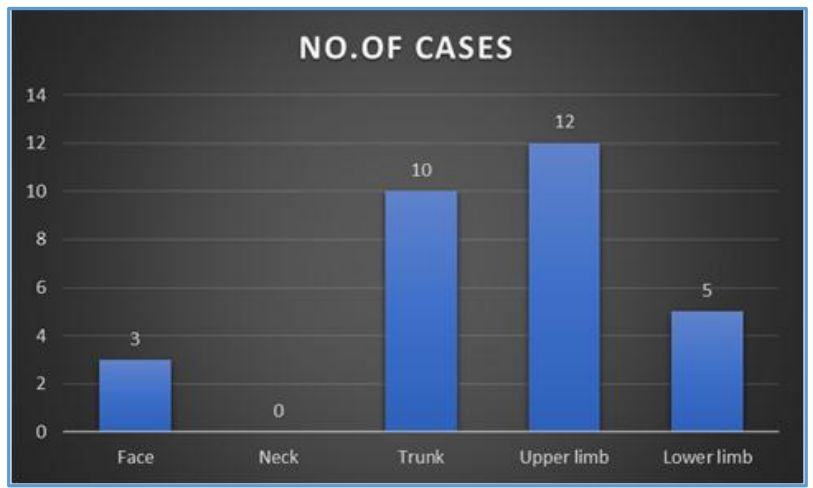

Figure 2. Site Distribution

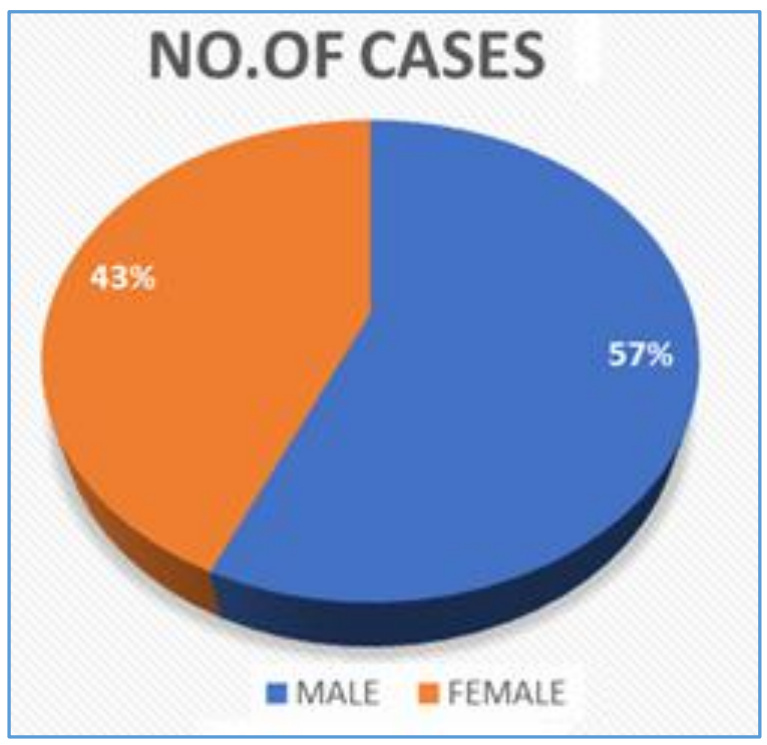

Figure 3. Sex Distribution

\section{CONCLUSION}

Linear lesions are diagnostic clues in many disorders. They help in elucidating the pathogenesis, as they give a clue to the spread of the disease. In this study, linear lesions occurring in paediatric age group. Lichen striatus was the most common condition encountered. To the best of my knowledge, there are much lesser case studies of linear lesions occurring in children as compared to the studies conducted in adults.

\section{REFERENCES}

[1] David K, Michael R, Sandra B, et al. Paediatric age categories to be used in differentiating between listing on a model essential medicines list for children. 20 April 2007.

[2] Tagra S, Talwar AK, Walia RLS. Lines of Blaschko. Indian J Dermatol Venereol Leprol 2005;71(1):57-9.

[3] Malvankar DD, Sacchidanand S, Mallikarjun M, et al. Linear lesions in dermatology. Indian J Dermatol Venereol Leprol 2011;77(6):722-6.

[4] Taieb A, el Youbi A, Grosshans E, et al. Lichen striatus: a Blaschko linear acquired inflammatory skin eruption. J Am Acad Dermatol 1991;25(4):637-42.

[5] Sittart JA, Pegas JR, Sant'Ana LA, et al. Lichen striatus: epidemiologic study. Med Cutan Ibero Lat Am 1989;17(1):19-21. 
[6] Hauber K, Rose C, Brocker EB, et al. Lichen striatus: clinical features and follow-up in 12 patients. European Journal of Dermatology 2000;10(7):536-9.

[7] Boyd AS, Neldner KH. Lichen planus. Journal of the American Academy of Dermatology 1991;25(4):593-619.

[8] Ireddy SG, Udbalkar SG. Study of Lichen planus and its different types and associated conditions. BMR Medicine 2014;1(1):1-11.

[9] Gurusamy L, Selvaraj U. Clinicopathological study of Lichen planus in a tertiary care center. Int J Sci Stud 2016;4(1):244-7.
[10] Metta AK, Ramachandra S, Sadath N, et al. Linear and whorled nevoid hypermelanosis in Three successive generations. Indian J Dermatol Venereol Leprol 2011;77(3):403.

[11] Shobha N, Taly AB, Sinha S, et al. Neurological pictures. Hypomelanosis of Ito. Journal of Neurology, Neurosurgery \& Psychiatry 2006;77(7): p. 873.

[12] Singh G, Yousuf Ali S. Paederus dermatitis. Indian J Dermatol Venereol Leprol 2007;73(1):13-5. 